\title{
Observation of a Universal Aggregation Mechanism and a Possible Phase Transition in Au Sputtered by Swift Heavy Ions
}

\author{
P. K. Kuiri, ${ }^{1}$ B. Joseph,${ }^{1, *}$ H. P. Lenka,${ }^{1}$ G. Sahu, ${ }^{1}$ J. Ghatak, ${ }^{1}$ D. Kanjilal, ${ }^{2}$ and D. P. Mahapatra ${ }^{1, \dagger}$ \\ ${ }^{1}$ Institute of Physics, Sachivalaya Marg, Bhubaneswar 751005, India \\ ${ }^{2}$ Inter University Accelerator Centre, Aruna Asaf Ali Marg, New Delhi 110067, India
}

\begin{abstract}
Two exponents, $\delta$, for size distribution of $n$-atom clusters, $Y(n) \sim n^{-\delta}$, have been found in Au clusters sputtered from embedded $\mathrm{Au}$ nanoparticles under swift heavy ion irradiation. For small clusters, below $12.5 \mathrm{~nm}$ in size, $\delta$ has been found to be $3 / 2$, which can be rationalized as occurring from a steady state aggregation process with size independent aggregation. For larger clusters, a $\delta$ value of $7 / 2$ is suggested, which might come from a dynamical transition to another steady state where aggregation and evaporation rates are size dependent. In the present case, the observed decay exponents do not support any possibility of a thermodynamic liquid-gas type phase transition taking place, resulting in cluster formation.
\end{abstract}

PACS numbers: 61.46.Df, 87.15.nr, 61.80.Jh, 68.37.Lp

The observation of emission of clusters of few atoms and molecules, from energetic ion impact was reported way back in 1958 [1]. This observation was quite surprising since the cluster binding energies are of the order of 1-2 eV, too small compared to the energies of the colliding ions. Since then there has been a lot of studies on the subject with an aim to understand the basic mechanisms behind cluster emission [2, 3, 4, 5, 6, 7]. In most cases, involving low energy ion impact [2, 3 , , 4], the cluster yield, $Y(n)$, as a function of number of constituent atoms, $n$, has been found to follow an inverse power law, $Y(n) \sim n^{-\delta}, \delta$ being a decay exponent. For small clusters with very few atoms, detected using time-of-flight, $\delta$ has been found to lie between 4 and 8 [2]. However, using transmission electron microscopy (TEM), it has recently been possible to study the size distribution of large clusters, collected on catcher foils, placed suitably during ion irradiations [4, 5].

On the theoretical side there is a shock-wave model [8] where overlapping collision cascades, from low energy heavy ion impact, can result in the production of shockwaves in a medium. These can propagate to the surface. In some cases they can result in emission of a chunk of material with essentially the same atomic coordination as the target. This mechanism, yields a value of $\delta$ close to 2. Sputtering data from Au thin films, irradiated using four different ions with energies in the range of 400 to $500 \mathrm{keV}$, have been shown to be in line with this [4]. Competing with the above model, there is also a thermodynamic model [9] where the cascade of atomic displacements, produced in the near surface region of a target, can thermalize and expand into vacuum. The temperature is supposed to go beyond the liquid-gas critical tem-

\footnotetext{
*Present address: Istituto Tecnologie Avanzate, Contrada Milo 91100, Trapani, Italy

${ }^{\dagger}$ Electronic mail: dpm@iopb.res.in
}

perature, $T_{C}$. Upon expansion and cooling the material can undergo a liquid-gas phase transition leading to cluster formation. In such a case the size distribution has been shown to follow a power law decay with a $\delta$ value close to $7 / 3$.

As compared to continuous media (films or bulk), irradiation of nm sized metal islands or metal nanoparticles (NPs), embedded in a matrix, with a possibility of melting and evaporation, form a different class of systems. In this letter, we show that the size distribution of clusters emitted from such a system, under swift heavy ion (SHI) irradiation, falls under a universal class of aggregation. These systems possess non-equilibrium steady state solutions of mass distributions in the form of inverse power laws. In all such cases there is a competition between aggregation and breakup or evaporation, a delicate balance between the two leading to a variety of steady state mass distributions [10]. Using a simple two-dimensional lattice model with jump between nearest sites and aggregation, Takayasu et al. [11] have shown that the asymptotic distribution of mass or size always follows a power law only with the injection of a unit mass (monomer) at each site. Without the injection of monomers the solution, in the infinite time limit, corresponds to an aggregate with all the particles sticking together. Later the analysis was extended to include both positive and negative values for the dynamical variable which was taken to be charge rather than mass [12]. The model included both injection and evaporation (through pair creation injection of unit positive and negative charges). The kinetics of aggregation were studied using a mean field theory. The system was found to reach a steady state with a charge distribution following a power law with $\delta=3 / 2$ [12]. This happens to be a very general case corresponding to a broad class of phenomena. As shown by Bonabeau et al. [13, 14], fish schools, with breakup and injection, show a similar aggregation, the size distribution showing a decay with $\delta=3 / 2$. This is seen even in economics related to distribution of wealth [15]. Such a result has also 
been obtained by Majumdar et al. [16] for aggregation in a mass conserving mean field type site-site interaction model. It has also been shown that small changes in the breakup parameters do not affect the decay exponent [14, 15]. There is however a cutoff size which depends upon the competition between aggregation and breakup, and the time scales associated with them.

Here we show, $\mathrm{Au}$ atoms sputtered by SHI from $\mathrm{Au}$ NPs follow a steady state aggregation as mentioned above. The observed size distribution is found to be in the form of a truncated power law with a $\delta$ value of $3 / 2$. Beyond a critical size of about $12.5 \mathrm{~nm}$ there is a drop off, which appears to be again in the form of another power law with a much larger exponent. Under the present irradiation conditions, the temperature of the NPs is known to go well above the vaporization temperature. But the size distribution shows power law decays with $\delta$ values of $3 / 2$ and $7 / 2$, quite different from $7 / 3$ as suggested for a liquid-gas type phase transition model [9]. Since the system indicates a steady state scenario there is no need to correct the size distribution against any breakup effects as applicable for cluster emission at lower irradiation energies [17]. The results also indicate the thermal spike production from electronic energy loss to be an essential requirement for the present observations.

For the present study, samples were prepared implanting $32 \mathrm{keV} \mathrm{Au}{ }^{-}$ions to a fluence of $4 \times 10^{16} \mathrm{~cm}^{-2}$ into silica glass substrates followed by annealing at $850^{\circ} \mathrm{C}$ in air for $1 \mathrm{~h}$. Hereafter, these will be called "targets". The $\mathrm{Au}$ implantation was carried out using a low energy negative ion implantation facility at the Institute of Physics (IOP), Bhubaneswar. For SHI, we have taken $\mathrm{Au}^{8+}$ ions at $100 \mathrm{MeV}$. Three of the targets were irradiated with SHI at normal incidence to fluences F2, F5, and F10 with values of $2 \times 10^{13}, 5 \times 10^{13}$, and $1 \times 10^{14}$ ions $\mathrm{cm}^{-2}$, respectively. These irradiations were carried out using the 16 MV Pelletron Accelerator at the Inter University Accelerator Centre (IUAC), New Delhi. For comparison, a fourth target was irradiated with $10 \mathrm{MeV} \mathrm{Au}^{4+}$ ions to a fluence as given by F10 using the 3 MV Pelletron accelerator at IOP, Bhubaneswar. In each case, both involving the low energy implantation (for target preparation) and the high energy target irradiations, the ion beams were raster scanned over an area of $1 \times 1 \mathrm{~cm}^{2}$ for uniform irradiation. During the irradiation of the targets, sputtered particles were collected using catcher foils (in the form of carbon coated $\mathrm{Cu}$ grids usually used for TEM studies) placed at a distance of $\sim 1 \mathrm{~cm}$ in front of the target at an angle of $\sim 15^{\circ}$ with the sample surface. All the $\mathrm{Au}$ implantation and irradiations were carried out at room temperature. The starting target and the catcher foils with collected particles were imaged using a JEOL 2010 UHR TEM operating at $200 \mathrm{kV}$. The Au content in the targets were checked before and after irradiation using Rutherford backscattering spectrometry (RBS) employing $1.35 \mathrm{MeV}^{4} \mathrm{He}^{+}$ions.
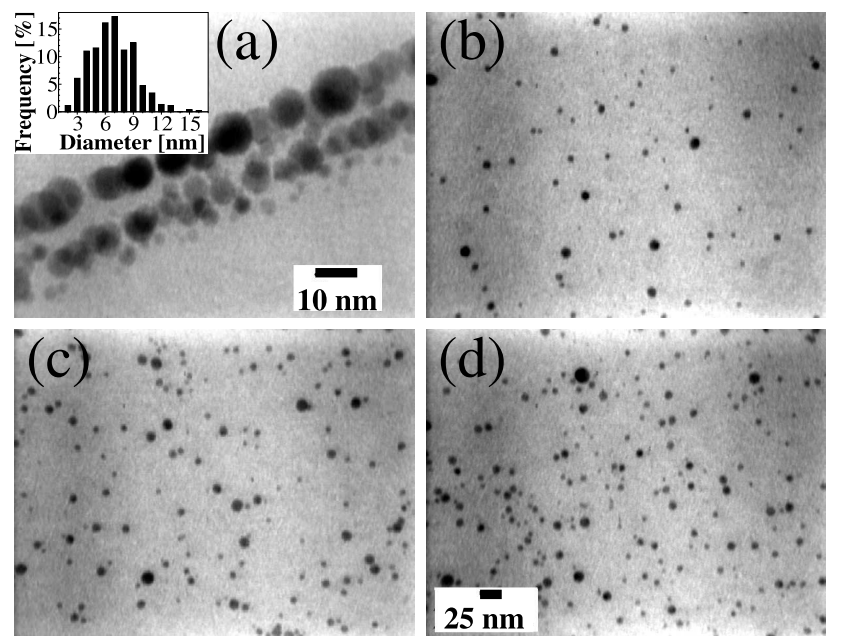

FIG. 1: (a) Bright-field cross-sectional TEM micrograph of the target showing Au NPs embedded in the matrix, before irradiation. Inset shows the size distribution of the Au NPs. (b) -(d) show plan-view TEM micrographs of the Au NPs on the catcher foils for SHI irradiations corresponding to the fluences of $2 \times 10^{13}, 5 \times 10^{13}$, and $1 \times 10^{14}$ ions $\mathrm{cm}^{-2}$, respectively. Note that (b) and (c) are having same scale as (d).

Bright-field cross-sectional TEM image [Fig 1 (a)] taken on the target shows a buried layer of spherical NPs with particle size decreasing from about 15 to $2 \mathrm{~nm}$, progressively with depth. Planar TEM micrographs of the particles collected on the catcher foils following SHI irradiations to fluences of F2, F5, and F10 are shown in Figs. 1(b), (c), and (d), respectively. The larger particles are noticeably darker in the micrograph indicating them to be 3-dimensional entities. The particles are seen to have sizes ranging from about 1 to $20 \mathrm{~nm}$. Figures 1(b)-(d) also show an increase in NP number density with increase in fluence.

From high resolution TEM and selected area electron diffraction measurements, the Au NPs in silica glass and on the catcher foils were found to be crystalline in nature with face centered cubic structure. Unlike the SHI irradiation no $\mathrm{Au} \mathrm{NP}$ was found on the catcher foil for irradiation at $10 \mathrm{MeV}$. RBS measurements, carried out on the target after $10 \mathrm{MeV} \mathrm{Au}$ irradiation, showed no $\mathrm{Au}$ loss in contrast to what is found after the SHI irradiation (Fig. 2). In the later case the Au loss is seen to increase with increase in fluence.

The size distributions of the NPs collected on the catcher foils, for various SHI fluences, are shown in Fig. 3 where the number of observed Au NPs of different sizes, $Y(n)$, has been plotted against $n$, the number of atoms in the $n$-atom cluster or NP. To generate these data, many TEM micrographs of the same frame size [as shown in Figs. 1(b)-[1(d)] were analyzed taking each NP to be spherical in shape. The number of atoms in an $n$-atom $\mathrm{NP}$ is estimated multiplying the volume of the NP by 


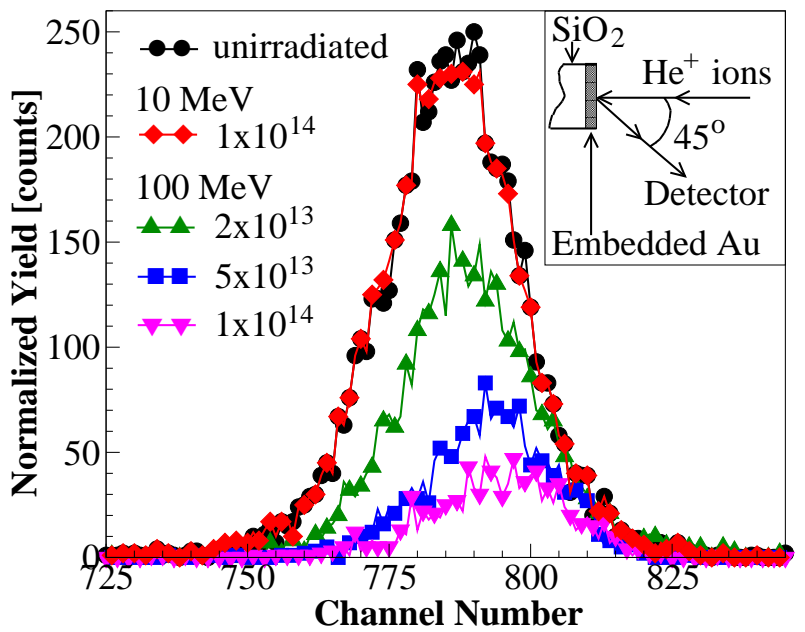

FIG. 2: (Color online) The Au part of the RBS spectra as measured on the targets before and after the irradiations. The irradiation fluences are in the unit of ions $\mathrm{cm}^{-2}$. Inset shows the experimental arrangement.

the number density of atoms in bulk Au. A total of 1313, 2009, and 2047 particles were considered for SHI fluences of F2, F5, and F10 respectively. Superimposed on the data is a straight line representing a power law distribution with a $\delta$ value of $3 / 2$ (dotted line). This is seen to agree with the data very well up to a cutoff size of about $12.5 \mathrm{~nm}$ (corresponding to about 62000 atoms) beyond which evaporation or breakup effects are dominant. This region corresponding to larger clusters and is affected by fluctuations because of progressively small number of particles observed. For this region we have also shown a power law decay with a $\delta$ value of $7 / 2$ (continuous line). The data points seem to follow this behavior. What is more important is that the data for three different fluences, taken on three different samples, show the same behavior. As shown earlier 18], SHI irradiation results in a change in the size distribution of the embedded Au NPs. But this does not seem to affect the size distribution of the sputtered $\mathrm{Au}$ NPs. In the following section we present a discussion on various aspects of the observed phenomenon and the conditions under which such aggregation takes place.

The first and formost requirement is the ejection of $\mathrm{Au}$ atoms from embedded $\mathrm{Au}$ NPs under SHI irradiation. This can happen due to the formation of localized inelastic thermal spikes [19] produced in the NPs resulting in their vaporization. In the inelastic thermal spikes model, passage of a SHI results in excitation and ionization of electrons in a cylindrical region around the ion path. This energy at first gets distributed into the electronic system through electron-electron interactions in a time scale of $\sim 10^{-13} \mathrm{~s}$. Electron-lattice interactions cause a part of this energy to go to lattice atoms resulting in a temperature rise. In case of small NPs the tempera-

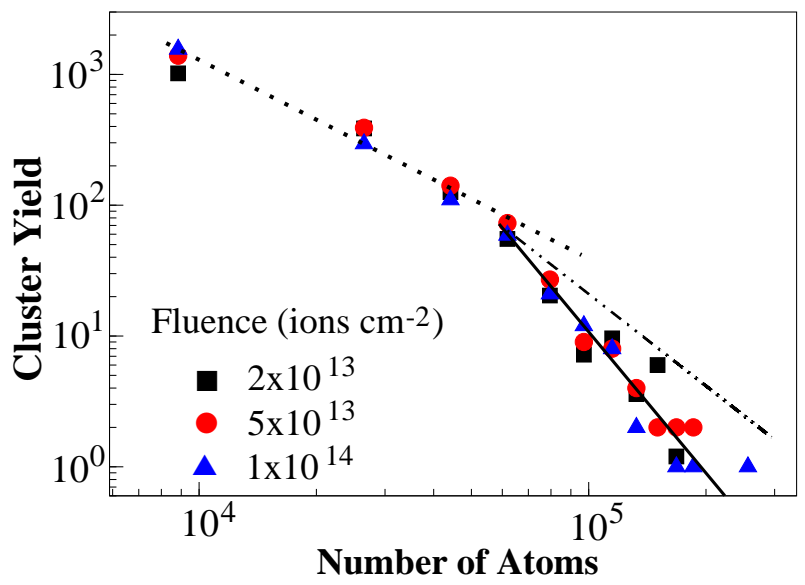

FIG. 3: (Color online) Size distribution in terms of the number of clusters of particular size (cluster yield) plotted against the number of atoms in the cluster. Dotted, dot-dashed, and continuous lines correspond to power law decays with $\delta$ values of $3 / 2,7 / 3$, and $7 / 2$, respectively.

ture of the thermal spike may go well above that required for vaporization because of the small volume. There is also no dissipation of heat into the insulating surrounding matrix. Simulations indicate this to be true leading to vaporization of smaller particles [20], some of which get attached to other NPs leading to Ostwald ripening. Although not shown here, cross-sectional TEM images taken on the targets, after SHI irradiations at various fluences, do show this. Such a phenomenon does not seem to happen with $10 \mathrm{MeV} \mathrm{Au}$ ions where electronic stopping $\left(2.5 \mathrm{keV} \mathrm{nm}^{-1}\right)$ is way below that at $100 \mathrm{MeV}(13.5$ $\left.\mathrm{keV} \mathrm{nm}{ }^{-1}\right)$. The vaporized material must also come out of the matrix for the observed aggregation to take place. In silica glass SHI irradiation can result in a melting of a cylindrical zone around the beam path which, because of the pressure imbalance [20], can result in a squeezing out of the evaporated $\mathrm{Au}$ atoms [18.

In such a case, it is not obvious that the expanding system could be in a thermalized state, with a temperature above a critical value, $T_{C}$, before going through a liquidgas phase transition as proposed by Urbassek [9]. In case it goes through such a transition, through the liquid gas co-existence region, there would be droplet formation. The size distribution of the sputtered clusters is then expected to show a power law decay with a $\delta$ value as given by Fisher's critical exponent, $\tau$, which lies between 2 and 2.5. For a Van der Waal's gas the exponent $\tau$ has a value of $7 / 3$. Compared to this, in the present case a $\delta$ value of $3 / 2$ has been obtained for smaller clusters which changes over to $7 / 2$ for larger ones. In fact there is no indication of an exponent close to $7 / 3$ which is also shown in Fig. 3 (dot-dashed line) for comparison. This rules out, any possible liquid-gas type phase transition taking place in the vaporized $\mathrm{Au}$ system resulting from SHI irradiation. 
On the other hand, exchange of particles between nucleation sites, within the framework of a mass-aggregation model, that takes diffusion, aggregation on contact, and dissociation, can result in a steady state aggregation process with a $\delta$ value of $3 / 2[16]$. What is more interesting is that the exponent $3 / 2$ appears in a variety of systems, constituting a universal class where mass plays the role of a control parameter. Mass conservation with steady injection of monomers is all that is required with other details of aggregation or breakup not being important. These results are also in disagreement with simulation results on cluster emission from Au NPs under self-ion irradiation at lower energies [21] where electronic stopping effects are neglected.

It is also important to understand the reason behind the cutoff observed in the cluster size distribution at about $12.5 \mathrm{~nm}$ and the change over taking place at that point. Such a cutoff can come from splitting of larger clusters [13]. But as long as aggregation and evaporation rates are independent of mass or size, the $\delta$ value, up to the cutoff, remains $3 / 2$. On the other hand a change over can occur when the aggregation and evaporation rates become mass dependent. As shown by Vigil et al. [22], for a critical value of the ratio of the two rates, there can be a transition between a steady state mass distribution and geletion (infinite mass aggregate). A power law decay, with a $\delta$ value of $7 / 2$, has been shown to occur at the transition point. It appears, in the present case, at cluster sizes greater than $12.5 \mathrm{~nm}$, somehow both the aggregation and evaporation rates become mass dependent leading to a phase transition from one steady state behavior with a $\delta$ value of $3 / 2$ to another with a $\delta$ value of $7 / 2$. At the moment it is not clear as to how such a transition takes place.

However, based on the present results, it is difficult to rule out the occurrence of a liquid-gas type phase transition in sputtered material for any general ion-target combination where the ion energy also plays a crucial role. In fact some experimental data do exist in support of the above model [23]. What has been shown here is the existence of a new mechanism of aggregation in sputtered particles, not shown earlier.

To conclude, swift heavy ion (100 $\left.\mathrm{MeV} \mathrm{Au}^{8+}\right)$ irradiation of $\mathrm{Au}$ NPs, embedded in silica glass, has been found to result in ejection of vaporized Au NPs which follow a rather universal aggregation mechanism occurring in nature. For smaller clusters, the size distribution shows a power law decay with a $\delta$ value of $3 / 2$ as observed in the steady state solution for non-equilibrium aggregation processes with a steady injection of monomers. For sizes greater than $12.5 \mathrm{~nm}$, there seems to be a change over to another steady state aggregation with a higher $\delta$ value indicating mass dependent effects coming into play. The results do not indicate any liquid-gas phase transition taking place in the sputtered $\mathrm{Au}$ upon cooling.

The authors wish to thank S. M. Bhattacharjee and
G. Tripathy of IOP, Bhubaneswar, for some very useful discussions and suggestions. We are also thankful to the Pelletron group of the accelerator facilities at IUAC, New Delhi and IOP, Bhubaneswar, in particular Mrs. Ramarani Das, for the help provided during ion irradiation experiments.

[1] R. E. Honing, J. Appl. Phys. 29, 549 (1958).

[2] C. Staudt, R. Heinrich, and A. Wucher, Nucl. Instrum. Methods Phys. Res. B 164/165, 677 (2000).

[3] C. Staudt and A. Wucher, Phys. Rev. B 66, 075419 (2002).

[4] L. E. Rehn, R. C. Birtcher, S. E. Donnelly, P. M. Baldo, and L. Funk, Phys. Rev. Lett. 87, 207601 (2001); L. E. Rehn, R. C. Birtcher, P. M. Baldo, A. W. McCormick, and L. Funk, Nucl. Instrum. Methods Phys. Res. B 212, 326 (2003).

[5] P. K. Kuiri, B. Joseph, J. Ghatak, H. P. Lenka, G. Sahu, B. S. Acharya, and D. P. Mahapatra, Nucl. Instrum. Methods Phys. Res. B 248, 25 (2006).

[6] P. K. Kuiri, J. Ghatak, B. Joseph, H. P. Lenka, G. Sahu, D. P. Mahapatra, A. Tripathi, D. Kanjilal, and N. C. Mishra, J. Appl. Phys. 101, 014313 (2007).

[7] B. Satpati, J. Ghatak, B. Joseph, T. Som, D. Kabiraj, B. N. Dev, and P. V. Satyam, Nucl. Instrum. Methods Phys. Res. B 244, 278 (2006).

[8] I. S. Bitensky and E. S. Parilis, Nucl. Instrum. Methods Phys. Res. B 21, 26 (1987).

[9] H. M. Urbassek, Nucl. Instrum. Methods Phys. Res. B 31, 541 (1988).

[10] W. White, J. Colloid Interface Sci. 87, 204 (1982).

[11] H. Takayasu, I. Nishikawa, and H. Tasaki, Phys. Rev. A 37, 3110 (1988).

[12] H. Takayasu, Phys. Rev. Lett. 63, 2563 (1989).

[13] E. Bonabeau, L. Dagorn, and P. Fréon, Proc. Natl. Acad. Sci. USA 96, 4472 (1999).

[14] E. Bonabeau and L. Dagorn, Phys. Rev. E 51, R5220 (1995).

[15] H. Yamamoto, T. Ohtsuki, A. Fujihara, and S. Tanimoto, J. Phys. Conference Series 31, 59 (2006).

[16] S. N. Majumdar, S. Krishnamurthy, and M. Barma, Phys. Rev. Lett. 81, 3691 (1998).

[17] K. O. E. Henriksson, K. Nordlund, and J. Keinonen, Phys. Rev. B 71, 014117 (2005).

[18] B. Joseph, J. Ghatak, H. P. Lenka, P. K. Kuiri, G. Sahu, N. C. Mishra, and D. P. Mahapatra, Nucl. Instrum. Methods Phys. Res. B 256, 659 (2007).

[19] M. Toulemonde, C. Trautmann, E. Balanzat, K. Hjort, and A. Weidinger, Nucl. Instrum. Methods Phys. Res. B 216, 1 (2004).

[20] C. D'Orléans, J. P. Stoquert, C. Estournés, C. Cerruti, J. J. Grob, J. L. Guille, F. Haas, D. Muller, and M. Richard-Plouet, Phys. Rev. B 67, 220101 (2003).

[21] R. Kissel and H. M. Urbassek, Nucl. Instrum. Methods Phys. Res. B 180, 293 (2001)

[22] R. D. Vigil, R. M. Ziff, and B. Lu, Phys. Rev. B 38, 942 (1988).

[23] H. T. Jonkman and J. Michl, J. Am. Chem. Soc. 103, 733 (1981); R. G. Orth, H. T. Jonkman, D. H. Powell, and J. Michl, J. Am. Chem. Soc. 103, 6026 (1981). 